\title{
ON UNITARY REPRESENTATIONS OF ALGEBRAIC GROUPS OVER LOCAL FIELDS
}

\author{
BACHIR BEKKA AND SIEGFRIED ECHTERHOFF
}

\begin{abstract}
Let $\mathbf{G}$ be an algebraic group over a local field $\mathbf{k}$ of characteristic zero. We show that the locally compact group $\mathbf{G}(\mathbf{k})$ consisting of the $\mathbf{k}$-rational points of $\mathbf{G}$ is of type I. Moreover, we complete Lipsman's characterization of the groups $\mathbf{G}$ for which every irreducible unitary representation of $\mathbf{G}(\mathbf{k})$ is a CCR representation and show at the same time that such groups belong to the family of trace class groups, recently studied by Deitmar and van Dijk.
\end{abstract}

\section{INTRODUCTION}

Given a second countable locally compact group $G$, a problem of major interest is to determine the unitary dual space $\widehat{G}$ of $G$, that is, the set of equivalence classes of irreducible unitary representations of $G$. The space $\widehat{G}$ carries a natural Borel structure, called the Mackey Borel structure, which is defined as follows (see [Mac57, §6] or [Dix77, §18.6]). For every $n \in\{1,2, \ldots, \infty\}$, let $\operatorname{Irr}_{n}(G)$ denote the space of all irreducible unitary representations of $G$ in a fixed Hilbert space $\mathcal{H}_{n}$ of dimension $n$. The set $\operatorname{Irr}_{n}(G)$ is equipped with the weakest Borel structure for which the functions $\pi \mapsto\langle\pi(g) \xi, \eta\rangle$ are measurable for all $\xi, \eta \in \mathcal{H}_{n}$; the disjoint union $\operatorname{Irr}(G)=\dot{\cup}_{n} \operatorname{Irr}_{n}(G)$ is endowed with the sum Borel structure of the Borel structures of the $\operatorname{Irr}_{n}(G)$ 's and $\widehat{G}$ with the quotient structure for the canonical surjective map $\operatorname{Irr}(G) \rightarrow \widehat{G}$.

A classification of $\widehat{G}$ is only possible if $\widehat{G}$ is countably separated, that is, if there exists a sequence of Borel subsets of $\widehat{G}$ which separates the points of $\widehat{G}$. By a result of Glimm (Gli61]), a group $G$ as above has this last property if and only if $G$ is of type I in the sense of the following definition.

Recall that a von Neumann algebra is a selfadjoint subalgebra of $\mathcal{L}(\mathcal{H})$ which is closed for the weak operator topology of $\mathcal{L}(\mathcal{H})$, where $\mathcal{H}$ is a Hilbert space. A von Neumann algebra $\mathcal{M}$ is a factor if the center of $\mathcal{M}$ consists of the scalar operators. A unitary representation $\pi$ of $G$ in a Hilbert space $\mathcal{H}$ is a factor representation if the von Neumann subalgebra $W_{\pi}^{*}$ of $\mathcal{L}(\mathcal{H})$ generated by $\pi(G)$ in $\mathcal{L}(\mathcal{H})$ is a factor.

Definition 1. A locally compact group $G$ is of type $\mathbf{I}$ if, for every factor representation $\pi$ of $G$, the factor $W_{\pi}^{*}$ is of type I, that is, $W_{\pi}^{*}$ is isomorphic to the

Received by the editors March 23, 2020.

2020 Mathematics Subject Classification. Primary 22D10, 22D25, 22E50, 20 G05.

The first author acknowledges the support by the ANR (French Agence Nationale de la Recherche) through the projects Labex Lebesgue (ANR-11-LABX-0020-01) and GAMME (ANR14-CE25-0004). This research was also funded by the Deutsche Forschungsgemeinschaft (DFG, German Research Foundation) under Germany's Excellence Strategy EXC 2044-390685587, Mathematics Münster: Dynamics-Geometry-Structure. 
von Neumann algebra $\mathcal{L}(\mathcal{K})$ for some Hilbert space $\mathcal{K}$; equivalently, if $\pi$ is equivalent to a multiple $n \sigma$ of an irreducible unitary representation $\sigma$ of $G$ for some $n \in\{1,2, \ldots, \infty\}$.

The main result of this note is to establish the type I property for algebraic groups. Let $\mathbf{K}$ be an algebraically closed field. A linear algebraic group over $\mathbf{K}$ is a Zariski closed subgroup of $G L_{n}(\mathbf{K})$ for some $n \geq 1$. If $\mathbf{k}$ is a subfield of $\mathbf{K}$ and if $\mathbf{G}$ is defined over $\mathbf{k}$, the group of $\mathbf{k}$-rational points of $\mathbf{G}$ is the subgroup $\mathbf{G}(\mathbf{k})=\mathbf{G} \cap G L_{n}(\mathbf{k})$. By a local field, we mean a non discrete locally compact field.

Theorem 2. Let $\mathbf{k}$ be a local field of characteristic 0 and let $\mathbf{G}$ be a linear algebraic group defined over $\mathbf{k}$. The locally compact group $\mathbf{G}(\mathbf{k})$ is of type $I$.

Some comments on Theorem 2 are in order.

Remark 3.

(i) The case where $\mathbf{G}$ is reductive is due to Harish-Chandra ( and to Bernstein (Ber74]) for non archimedean $\mathbf{k}$.

(ii) In the case $\mathbf{k}=\mathbf{R}$, Theorem 2 is a result due to Dixmier ([Dix57]).

(iii) Duflo ([Duf82] describes a general procedure to determine the unitary dual for a group $\mathbf{G}(\mathbf{k})$ as in Theorem 2, As is known to experts, one could derive the fact that $\mathbf{G}(\mathbf{k})$ is type I from Duflo's results, provided Bernstein's result is extended to certain finite central extensions of reductive groups. This note offers a direct approach, independent of [Duf82. Indeed, our proof of Theorem 2 closely follows the main strategy in the case $\mathbf{k}=\mathbf{R}$ from Dix57. (see also Puk99]). In particular, we obtain a new proof of the known fact (Dix59], Kir62], Moo65]) that $\mathbf{G}(\mathbf{k})$ is of type I when $\mathbf{G}$ is unipotent. As a necessary preliminary step, we establish the extension mentioned above of Bernstein's result to certain finite central extensions of reductive groups (see Subsection 2.6).

(iv) Assume that $\mathbf{k}$ is a local field of positive characteristic. Then $\mathbf{G}(\mathbf{k})$ is still of type I when $\mathbf{G}$ is reductive (Ber74]). To extend the result to an arbitrary $\mathbf{G}$, the major difficulty to overcome is to show that $\mathbf{G}(\mathbf{k})$ is of type I when $\mathbf{G}$ is unipotent, a fact which - to our knowledge - is unknown.

Let $G$ be a locally compact group. Recall that, if $\pi$ is a unitary representation of $G$ on a Hilbert space $\mathcal{H}$, then $\pi$ extends to a representation by bounded operators on $\mathcal{H}$ of the convolution algebra $C_{c}(G)$ of continuous functions with compact support on $G$, defined by

$$
\pi(f)=\int_{G} f(x) \pi(x) d x \quad \text { for all } \quad f \in C_{c}(G),
$$

where $d x$ denotes a Haar measure on $G$. The representation $\pi$ is said to be a CCR representation if $\pi(f)$ is a compact operator for every $f \in C_{c}(G)$. The group $G$ is a CCR group if every $\pi \in \widehat{G}$ is a CCR representation. It follows from [Dix77, Theorem 9.1] that every CCR group is type I.

Let $C_{c}^{\infty}(G)$ be the space of test functions on $G$ as defined in Bru61. Following DvD16, we say that the representation $\pi$ of $G$ is of trace class if $\pi(f)$ is a trace class operator for every $f \in C_{c}^{\infty}(G)$. The group $G$ is called a trace class group, if every irreducible unitary representation of $G$ is trace class. Since $C_{c}^{\infty}(G)$ is dense in $L^{1}(G, d x)$, it is clear that a trace class group is CCR, and hence of type I. 
Let $\mathbf{G}$ and $\mathbf{k}$ be as in Theorem 2. It is known that $\mathbf{G}(\mathbf{k})$ is a CCR group, if $\mathbf{G}$ is reductive (see [HC53, and Ber74]) or if $\mathbf{G}$ is unipotent (see Dix59, [Kir62, Moo65]). This result has been strengthened in DvD16] by showing that $\mathbf{G}(\mathbf{k})$ is a trace class group in these cases.

Let $\mathbf{U}$ be the unipotent radical of $\mathbf{G}$ and $\mathbf{L}$ a Levi subgroup defined over $\mathbf{k}$; so, we have a semi-direct product decomposition $\mathbf{G}=\mathbf{L} \mathbf{U}$. Let

$$
\mathbf{M}=\{g \in \mathbf{L} \mid g u=u g \quad \text { for all } u \in U\} .
$$

Then $\mathbf{M}$ is an algebraic normal subgroup of $\mathbf{L}$ defined over $\mathbf{k}$. Lipsman ([Lip75]) showed that, if $\mathbf{G}(\mathbf{k})$ is $\mathrm{CCR}$, then $\mathbf{L}(\mathbf{k}) / \mathbf{M}(\mathbf{k})$ is compact. Our second result concerns the converse statement (see also the comment immediately after Theorem 3.1 in Lip75).

Theorem 4. Let $\mathbf{G}$ and $\mathbf{k}$ be as in Theorem 2 , Let $\mathbf{L}$ and $\mathbf{M}$ be as above. The following properties are equivalent:

(i) $\mathbf{G}(\mathbf{k})$ is a CCR group;

(ii) $\mathbf{L}(\mathbf{k}) / \mathbf{M}(\mathbf{k})$ is compact;

(iii) $\mathbf{G}(\mathbf{k})$ is trace class.

Theorem 4 generalizes several results from vD19] and provides a positive solution to a much stronger version of Conjecture 14.1 stated there.

\section{SOME PRELIMINARY RESULTS}

2.1. Projective representations. Let $G$ be a second countable locally compact group. We will need the notion of a projective representation of $G$. For all what follows, we refer to Mac58.

Let $\mathcal{H}$ be a Hilbert space. Recall that a map $\pi: G \rightarrow U(\mathcal{H})$ from $G$ to the unitary group of $\mathcal{H}$ is a projective representation of $G$ if the following holds:

(i) $\pi(e)=I$,

(ii) for all $x, y \in G$, there exists $\omega(x, y) \in \mathbf{T}$ such that

$$
\pi(x y)=\omega(x, y) \pi(x) \pi(y),
$$

(iii) the map $g \mapsto\langle\pi(g) \xi, \eta\rangle$ is Borel for all $\xi, \eta \in \mathcal{H}$.

The map $\omega: G \times G \rightarrow \mathbf{T}$ has the following properties:

(iv) $\omega(x, e)=\omega(e, x)$ for all $x \in G$,

(v) $\omega(x y, z) \omega(x, y)=\omega(x, y z) \omega(y, z)$ for all $x, y, z \in G$.

The set $Z^{2}(G, \mathbf{T})$ of all Borel maps $\omega: G \times G \rightarrow \mathbf{T}$ with properties (iv) and (v) is an abelian group for the pointwise product.

For a given $\omega \in Z^{2}(G, \mathbf{T})$, a map $\pi: G \rightarrow U(\mathcal{H})$ with properties (i), (ii) and (iii) as above is called an $\omega$-representation of $G$.

For $i=1,2$, let $\omega_{i}, \in Z^{2}(G, \mathbf{T})$ and $\pi_{i}$ an $\omega_{i}$-representation of $G$ on a Hilbert space $\mathcal{H}_{i}$. Then $\pi_{1} \otimes \pi_{2}$ is an $\omega_{1} \omega_{2}$-representation of $G$ on the Hilbert space $\mathcal{H}_{1} \otimes \mathcal{H}_{2}$. In particular, if $\omega_{2}=\omega_{1}^{-1}$, then $\pi_{1} \otimes \pi_{2}$ is an ordinary representation of $G$.

Every projective unitary representation of $G$ can be lifted to an ordinary unitary representation of a central extension of $G$. More precisely, for $\omega \in Z^{2}(G, \mathbf{T})$, let $S$ be the closure in $\mathbf{T}$ of the subgroup generated by the image of $\omega$; define a group $G^{\omega}$ with underlying set $S \times G$ and multiplication $(s, x)(t, y)=(s t \omega(x, y), x y)$. Equipped with a suitable topology, $G^{\omega}$ is a locally compact group. Let $\pi: G \rightarrow U(\mathcal{H})$ be an $\omega$-representation of $G$. Then $\pi^{0}: G^{\omega} \rightarrow U(\mathcal{H})$, defined by $\pi^{0}(s, x)=s \pi(x)$ is an 
ordinary representation of $G^{\omega}$; moreover, the map $\pi \rightarrow \pi^{0}$ is a bijection between the $\omega$-representations of $G$ and the representations of $G^{\omega}$ for which the restriction to the central subgroup $S \times\{e\}$ is a multiple of the one dimensional representation $(s, e) \mapsto s$.

A projective representation $\pi$ of $G$ on $\mathcal{H}$ is irreducible (respectively, a factor representation) if the only closed $\pi(G)$-invariant subspaces of $\mathcal{H}$ are $\{0\}$ and $\mathcal{H}$ (respectively, if the von Neumann algebra generated by $\pi(G)$ is a factor). A factor projective representation $\pi$ is of type $I$, if the factor generated by $\pi(G)$ is of type $I$.

2.2. Factor representations. Let $G$ be a second countable locally compact group. Let $N$ be a type I closed normal subgroup of $G$. Then $G$ acts by conjugation on $\widehat{N}$ : for $\pi \in \widehat{N}$, the conjugate representation $\pi^{g} \in \widehat{N}$ is defined by $\pi^{g}(n)=\pi\left(g n g^{-1}\right)$, for $g \in G, n \in N$.

Let $\pi \in \widehat{N}$. The stabilizer

$$
G_{\pi}=\left\{g \in G: \pi^{g} \text { is equivalent to } \pi\right\}
$$

of $\pi$ is a closed subgroup of $G$ containing $N$. There exists a projective unitary representation $\tilde{\pi}$ of $G_{\pi}$ on $\mathcal{H}$ which extends $\pi$. Indeed, for every $g \in G_{\pi}$, there exists a unitary operator $\tilde{\pi}(g)$ on $\mathcal{H}$ such that

$$
\pi^{g}(n)=\widetilde{\pi}(g) \pi(n) \widetilde{\pi}(g)^{-1} \quad \text { for all } \quad n \in N .
$$

One can choose $\widetilde{\pi}(g)$ such that $g \mapsto \widetilde{\pi}(g)$ is an $\omega \circ(p \times p)$-representation of $G_{\pi}$ which extends $\pi$ for some $\omega \in Z^{2}(G / N, \mathbf{T})$, where $p: G \rightarrow G / N$ is the canonical projection (see Theorem 8.2 in Mac58]).

The normal subgroup $N$ is said to be regularly embedded in $G$ if the quotient space $\widehat{N} / G$, equipped with the quotient Borel structure inherited from $\widehat{N}$, is a countably separated Borel space.

The following result is part of the so-called Mackey machine; it is a basic tool which reduces the determination of the factor (or irreducible) representations of $G$ to the determination of the factor (or irreducible) representations of subgroups of $G$. The proof is a direct consequence of Theorems 8.1, 8.4 and 9.1 in Mac58.

Theorem 5. Let $N$ be a closed normal subgroup of $G$ and denote by $p: G \rightarrow G / N$ the canonical projection. Assume that $N$ is of type $I$ and is regularly embedded in $G$.

(i) Let $\pi \in \widehat{N}$ and $\pi^{\prime}$ a factor representation of $G_{\pi}$ such that the restriction of $\pi^{\prime}$ to $N$ is a multiple of $\pi$. Then the induced representation $\operatorname{Ind}_{G_{\pi}}^{G} \pi^{\prime}$ is a factor representation. Moreover, $\operatorname{Ind}_{G_{\pi}}^{G} \pi^{\prime}$ is of type $I$ if and only if $\pi^{\prime}$ is of type $I$.

(ii) Every factor representation of $G$ is equivalent to a representation of the form $\operatorname{Ind}_{G_{\pi}}^{G} \pi^{\prime}$ as in (i).

(iii) Let $\pi \in \widehat{N}$ and assume that $G=G_{\pi}$. Let $\omega \in Z^{2}(G / N, \mathbf{T})$ and $\tilde{\pi}$ an $\omega \circ(p \times$ $p)$-representation of $G$ which extends $\pi$. Then every factor representation $\pi^{\prime}$ of $G$ such that the restriction of $\pi^{\prime}$ to $N$ is a multiple of $\pi$ is equivalent to a representation of the form $\sigma \otimes \widetilde{\pi}$, where $\sigma$ is a factor $\omega^{-1}$-representation of $G / N$ lifted to $G$. Moreover, $\pi^{\prime}$ is of type $I$ if and only if $\sigma$ is of type $I$. 
We will need the well-known fact that being of type I is inherited from cocompact normal subgroups; for the sake of completeness, we reproduce a short proof modeled after [Puk99].

Proposition 6. Let $N$ be a normal subgroup of $G$. Assume that $N$ is of type $I$ and that $G / N$ is compact. Then $G$ is of type $I$.

Proof. Since $N$ is of type I, the Borel space $\widehat{N}$ is countably separated, by Glimm's result (mentioned in the introduction). The action of $G$ on $\widehat{N}$ factorizes to an action of the compact group $K:=G / N$.

We claim that $\widehat{N} / K$ is countably separated, that is, $N$ is regularly embedded in $G$. Indeed, by a theorem of Varadarajan (see Zim84, 2.1.19]), there exists a compact metric space $X$ on which $K$ acts by homeomorphisms and an injective $K$-equivariant Borel map $\widehat{N} \rightarrow X$. Since $K$ is compact, $X / K$ is easily seen to be countably separated and the claim follows.

Let $\pi \in \widehat{N}$ and $\sigma$ a projective factor representation of $G_{\pi} / N$. Then $\sigma$ lifts to an ordinary representation $\widetilde{\sigma}$ of a central extension $\widetilde{G_{\pi}}$ of $G_{\pi} / N$ by a closed subgroup of T. Since $G_{\pi} / N$ is compact, $\widetilde{G_{\pi}}$ is compact and therefore of type I. So, $\widetilde{\sigma}$ and hence $\sigma$ is of type I. Theorem 5 shows that $G$ is of type I.

We will use the following consequence of Proposition 6 in the proof of Theorem 4

Corollary 7. Let $G$ and $N$ be as in Proposition 6 . For every $\rho \in \widehat{G}$, there exists a representation $\pi \in \widehat{N}$ such that $\rho$ is a subrepresentation of $\operatorname{Ind}_{N}^{G} \pi$.

Proof. Let $\rho \in \widehat{G}$. By Proposition [6] $N$ is regularly embedded. Hence, by Theorem 5] there exist $\pi \in \widehat{N}$ and $\sigma \in \widehat{G}_{\pi}$ such that $\left.\sigma\right|_{N}$ is a multiple of $\pi$ and such that $\rho \simeq \operatorname{Ind}_{G_{\pi}}^{G} \sigma$.

As is well-known (see for instance [BdlHV08, Proposition E.2.5]),

$$
\operatorname{Ind}_{N}^{G_{\pi}}\left(\left.\sigma\right|_{N}\right) \simeq \sigma \otimes \operatorname{Ind}_{N}^{G_{\pi}} 1_{N}
$$

that is,

$$
\operatorname{Ind}_{N}^{G_{\pi}}\left(\left.\sigma\right|_{N}\right) \simeq \sigma \otimes \lambda_{G_{\pi} / N}
$$

where $\lambda_{G_{\pi} / N}$ is the regular representation of $G_{\pi} / N$ lifted to $G_{\pi}$. Since $G_{\pi} / N$ is compact, $\lambda_{G_{\pi} / N}$ contains the trivial representation $1_{G_{\pi}}$. It follows that $\operatorname{Ind}_{N}^{G_{\pi}}\left(\left.\sigma\right|_{N}\right)$ contains $\sigma$. Since $\left.\sigma\right|_{N}$ is multiple of $\pi$ and since $\sigma$ is irreducible, we see that $\sigma$ is contained $\operatorname{Ind}_{N}^{G_{\pi}} \pi$. Induction by stages shows then that $\rho \simeq \operatorname{Ind}_{G_{\pi}}^{G} \sigma$ is contained $\operatorname{in} \operatorname{Ind}_{G_{\pi}}^{G}\left(\operatorname{Ind}_{N}^{G_{\pi}} \pi\right) \simeq \operatorname{Ind}_{N}^{G} \pi$.

2.3. Actions of algebraic groups. Assume now that $\mathbf{k}$ is a local field of characteristic 0 and $\mathbf{G}$ a linear algebraic group defined over $\mathbf{k}$. If $V$ is an algebraic variety defined over $\mathbf{k}$, then the set $V(\mathbf{k})$ of $\mathbf{k}$-rational points in $V$ has also a locally compact topology, which we call the Hausdorff topology.

The following well-known result (see [Zim84, 3.1.3]) is a crucial tool for the sequel. We indicate briefly the main steps in its proof.

Theorem 8. Let $\mathbf{G} \times V \rightarrow V$ be a $\mathbf{k}$-rational action of $\mathbf{G}$ on an algebraic variety $V$ defined over $\mathbf{k}$. Then every $\mathbf{G}(\mathbf{k})$-orbit in $V(\mathbf{k})$ is open in its closure for the Hausdorff topology. 
Proof. Let $W$ be a G-orbit in $V$. Then $W$ is open in its closure for the Zariski topology (see [Bor91, 1.8]). This implies that $W(\mathbf{k})$ is open in its closure for the Hausdorff topology. By [BS64, 6.4], there are only finitely many $\mathbf{G}(\mathbf{k})$-orbits contained in $W(\mathbf{k})$ and the claim follows.

We will use the previous theorem in the case of a linear representation of $\mathbf{G}$. More precisely, let $\mathbf{K}$ be an algebraic closure of $\mathbf{k}$ and let $V$ be a finite dimensional vector space over $\mathbf{K}$, equipped with a k-structure $V_{\mathbf{k}}$. Let $\rho: \mathbf{G} \rightarrow G L(V)$ be a krational representation of $\mathbf{G}$. Consider the dual adjoint $\rho^{*}$ of $\mathbf{G}$ on the dual vector space $V^{*}=\operatorname{Hom}(V, \mathbf{K})$, equipped with the $\mathbf{k}$-structure $V_{\mathbf{k}}^{*}=\operatorname{Hom}\left(V_{\mathbf{k}}, \mathbf{k}\right)$. Then $\rho^{*}: \mathbf{G} \rightarrow G L\left(V^{*}\right)$ is a k-rational representation of $\mathbf{G}$. Fix a non-trivial unitary character $\varepsilon \in \widehat{\mathbf{k}}$ of the additive group of $\mathbf{k}$. The map $\Phi: V_{\mathbf{k}}^{*} \rightarrow \widehat{V_{\mathbf{k}}}$, given by

$$
\Phi(f)(v)=\varepsilon(f(v)) \quad \text { for all } f \in V^{*} \text { and } \quad v \in V_{\mathbf{k}},
$$

is an isomorphism of topological groups (see [Wei64, Chap.II, §5]). The group $\mathbf{G}(\mathbf{k})$ acts on $V_{\mathbf{k}}, V_{\mathbf{k}}^{*}$ and $\widehat{V_{\mathbf{k}}}$; the map $\Phi$ is $\mathbf{G}(\mathbf{k})$-equivariant. Therefore, the following corollary is a direct consequence of Theorem 8 .

Corollary 9. Let $\rho: \mathbf{G} \rightarrow G L(V)$ be a $\mathbf{k}$-rational representation of $\mathbf{G}$ as above. Every $\mathbf{G}(\mathbf{k})$-orbit in $\widehat{V_{\mathbf{k}}}$ is open in its closure.

Let $\mathbf{U}$ be the unipotent radical of $\mathbf{G}$ and $\mathfrak{u}$ its Lie algebra. Then $\mathbf{U}$ is an algebraic subgroup of $\mathbf{G}$ defined over $\mathbf{k}$ and the exponential map exp : $\mathfrak{u} \rightarrow \mathbf{U}$ is an isomorphism of $\mathbf{k}$-varieties; moreover, $\mathfrak{m} \rightarrow \exp \mathfrak{m}$ is a bijection between Lie subalgebras of $\mathfrak{u}$ and (connected) algebraic subgroups of $\mathbf{U}$.

The Lie algebra $\mathfrak{u}$ has a $\mathbf{k}$-structure $\mathfrak{u}_{\mathbf{k}}$ which is the Lie $\mathbf{k}$-subalgebra of $\mathfrak{u}$ for which $\exp : \mathfrak{u}_{\mathbf{k}} \rightarrow \mathbf{U}(\mathbf{k})$ is a bijection.

The action of $\mathbf{G}$ by conjugation on $\mathbf{U}$ induces an action, called the adjoint representation Ad : $\mathbf{G} \rightarrow G L(\mathfrak{u})$, which is a k-rational representation by automorphisms of the Lie algebra $\mathfrak{u}$ for which $\exp : \mathfrak{u} \rightarrow \mathbf{U}$ is $\mathbf{G}$-equivariant. The map $\mathfrak{m} \rightarrow \exp \mathfrak{m}$ is a bijection between $\operatorname{Ad}(\mathbf{G})$-invariant ideals of $\mathfrak{u}$ and algebraic normal subgroups of $\mathbf{G}$ contained in $\mathbf{U}$.

Lemma 10. Let $\mathbf{M}$ be an abelian algebraic normal subgroup of $\mathbf{G}$ contained in $\mathbf{U}$. Then $\mathbf{M}(\mathbf{k})$ is regularly embedded in $\mathbf{G}(\mathbf{k})$.

Proof. By an elementary argument (see [Zim84, 2.1.12]), it suffices to show that every $\mathbf{G}(\mathbf{k})$-orbit in $\widehat{\mathbf{M}(\mathbf{k})}$ is open in its closure. Let $\mathfrak{m}$ be the $\operatorname{Ad}(\mathbf{G})$-invariant ideal of $\mathfrak{u}$ corresponding to $\mathbf{M}$ and set $\mathfrak{m}_{\mathbf{k}}=\mathfrak{m} \cap \mathfrak{u}_{\mathbf{k}}$. Since $\mathbf{M}$ is abelian, exp : $\mathfrak{m}_{\mathbf{k}} \rightarrow \mathbf{M}(\mathbf{k})$ is an isomorphism of topological groups. So, it suffices to show that every $\mathbf{G}(\mathbf{k})$ orbit in $\widehat{\mathfrak{m}_{\mathbf{k}}}$ is open in its closure. This is indeed the case by Corollary 9 ,

2.4. Heisenberg groups and Weil representation. Let $\mathbf{k}$ be a field of characteristic 0 and let $n \geq 1$ be an integer. The Heisenberg group $H_{2 n+1}(\mathbf{k})$ is the nilpotent group with underlying set $\mathbf{k}^{2 n} \times \mathbf{k}$ and product

$$
((x, y), s)\left(\left(x^{\prime}, y^{\prime}\right), t\right)=\left(\left(x+x^{\prime}, y+y^{\prime}\right), s+t+\frac{1}{2} \beta\left((x, y),\left(x^{\prime}, y^{\prime}\right)\right)\right),
$$

for $(x, y),\left(x^{\prime}, y^{\prime}\right) \in \mathbf{k}^{2 n}, s, t \in \mathbf{k}$, where $\beta$ is the standard symplectic form on $\mathbf{k}^{2 n}$. The group $H_{2 n+1}(\mathbf{k})$ is the group of $\mathbf{k}$-rational points of a unipotent algebraic group $H_{2 n+1}$ defined over $\mathbf{k}$. Its Lie algebra $\mathfrak{h}_{2 n+1}$ has a basis $\left\{X_{1}, \ldots, X_{n}, Y_{1}, \ldots, Y_{n}, Z\right\}$ with non trivial commutators $\left[X_{i}, Y_{i}\right]=Z$ for all $i=1, \ldots, n$. The center of $\mathfrak{h}_{2 n+1}$ 
is equal to the commutator subalgebra $\left[\mathfrak{h}_{2 n+1}, \mathfrak{h}_{2 n+1}\right]$ and is spanned by $Z$. This last property characterizes $H_{2 n+1}$; for the proof, see [Dix57, Lemme 4].

Lemma 11. Let $\mathbf{U}$ be a unipotent algebraic group defined over $\mathbf{k}$ with Lie algebra $\mathfrak{u}$. Assume that the center $\mathfrak{z}$ of $\mathfrak{u}$ equals $[\mathfrak{u}, \mathfrak{u}]$ and that $\operatorname{dim} \mathfrak{z}=\operatorname{dim}([\mathfrak{u}, \mathfrak{u}])=1$. Then $\mathbf{U}$ is isomorphic to $H_{2 n+1}$ over $\mathbf{k}$ for some $n \geq 1$.

The symplectic group $S p_{2 n}$, which is the isometry group of $\beta$, acts by rational automorphisms of $H_{2 n+1}$, given by

$$
\varphi_{g}((x, y), t)=(g(x, y), t) \quad \text { for all } \quad g \in S p_{2 n},((x, y), t) \in H_{2 n+1} .
$$

Let $\operatorname{Aut}_{c}\left(H_{2 n+1}\right)$ be the group of automorphisms of $H_{2 n+1}$ which acts trivially on the center of $H_{2 n+1}$. Observe that $\varphi_{g} \in \operatorname{Aut}_{c}\left(H_{2 n+1}\right)$ for every $g \in S p_{2 n}$ and that $I_{h} \in \operatorname{Aut}_{c}\left(H_{2 n+1}\right)$ for every $h \in H_{2 n+1}$, where $I_{h}$ is the inner automorphism given by $h$. The following proposition is proved in [Fol89, 1.22] in the case $\mathbf{k}=\mathbf{R}$; however, its proof is valid in our setting.

Proposition 12. Every automorphism in $\operatorname{Aut}_{c}\left(H_{2 n+1}\right)$ can be uniquely written as the product $\varphi_{g} \circ I_{h}$ for $g \in S p_{2 n}$ and $h \in H_{2 n+1}$. So, Aut ${ }_{c}\left(H_{2 n+1}\right)$ can be identified with the semi-direct product $S p_{2 n} \ltimes H_{2 n+1}$.

The following facts about the irreducible representations of $H_{2 n+1}(\mathbf{k})$ and associated metaplectic (or oscillator) representations of $S p_{2 n}(\mathbf{k})$ will play a crucial role in the sequel; for more details, see Wei64.

Theorem 13. Denote by $Z$ the center of $H_{2 n+1}(\mathbf{k})$ and let $\chi \in \widehat{Z}$ be a non trivial character of $Z$.

(i) (Stone-von Neumann) There exists, up to equivalence, a unique irreducible unitary representation $\pi_{\chi}$ of $H_{2 n+1}(\mathbf{k})$ such that $\left.\pi_{\chi}\right|_{Z}$ is a multiple of $\chi$.

(ii) (Segal-Shale-Weil) The representation $\pi_{\chi}$ extends to a unitary representation of $\widetilde{S p_{2 n}(\mathbf{k})} \ltimes H_{2 n+1}(\mathbf{k})$, where $\widetilde{S p_{2 n}(\mathbf{k})}$ is a twofold cover of $S p_{2 n}(\mathbf{k})$, called the metaplectic group.

2.5. Large compact subgroups and admissible representations. Let $G$ be a locally compact group. We introduce a few notions from [War72, 7.5].

Definition 14. Let $K$ be a compact subgroup of $G$.

(i) $K$ is said to be large in $G$ if, for every $\sigma \in \widehat{K}$,

$$
\sup _{\pi \in \widehat{G}} m\left(\sigma,\left.\pi\right|_{K}\right)<+\infty,
$$

where $m\left(\sigma,\left.\pi\right|_{K}\right)$ is the multiplicity of $\sigma$ in $\left.\pi\right|_{K}$.

(ii) $K$ is said to be uniformly large in $G$, if there exists an integer $M$ such that, for every $\sigma \in \widehat{K}$,

$$
\sup _{\pi \in \widehat{G}} m\left(\sigma,\left.\pi\right|_{K}\right) \leq M \operatorname{dim} \sigma,
$$

Proposition 15. Let $G$ be a second countable locally compact group.

(i) Assume that $G$ contains a large compact subgroup. Then $G$ is $C C R$.

(ii) Assume that $G$ contains a uniformly large compact subgroup. Then $G$ is trace class. 
Proof. Item (i) is well known (see War72, Theorem 4.5.7.1]).

Assume that $G$ contains a uniformly large compact subgroup. Let $f \in C_{c}(G)$ and $\pi \in \widehat{G}$. Then $\pi(f)$ is a Hilbert-Schmidt operator ([War72, Theorem 4.5.7.4]). It follows from [DvD16, Proposition 1.6] that $\pi(f)$ is a trace class operator if $f \in$ $C_{c}^{\infty}(G)$.

We introduce further notions in the context of totally disconnected groups.

\section{Definition 16.}

(i) A representation $\pi: G \rightarrow G L(V)$ in a complex vector space $V$ is said to be smooth if the stabilizer in $G$ of every $v \in V$ is open.

(ii) A smooth representation or unitary representation $(\pi, V)$ of $G$ is admissible if the space $V^{K}$ of $K$-fixed vectors in $V$ is finite dimensional, for every compact open subgroup $K$ of $G$.

(iii) $G$ is said to have a uniformly admissible smooth dual (respectively, unitary dual) if, for every compact open subgroup $K$ of $G$, there exists a constant $N(K)$ such that $\operatorname{dim} V^{K} \leq N(K)$, for every irreducible smooth (respectively, irreducible unitary) representation $(\pi, V)$ of $G$.

Let $G$ be a totally disconnected locally compact group. For a compact open subgroup $K$ of $G$, let $\mathcal{H}(G, K)$ be the convolution algebra of continuous functions on $G$ which are bi-invariant under $K$. The algebra $\mathcal{H}(G, K)$ is a *-algebra, for the involution given by

$$
f^{*}(g)=\Delta(g) \overline{f\left(g^{-1}\right)} \quad \text { for all } \quad f \in \mathcal{H}(G, K), g \in G,
$$

where $\Delta$ is the modular function of $G$. Observe that

$$
\mathcal{H}(G, K)=e_{K} * C_{c}(G) * e_{K},
$$

where $e_{K}=\frac{1}{\mu(K)} \mathbf{1}_{K}$ and $\mu$ is a Haar measure on $G$.

Let $(\pi, V)$ be a smooth (respectively, unitary) representation of $G$ and let $K$ be a compact open subgroup of $G$. A representation (respectively, a $*$-representation) $\pi_{K}$ of $\mathcal{H}(G, K)$ is defined on $V^{K}$ by

$$
\pi_{K}(f) v:=\int_{G} f(g) \pi(g) v d \mu(g) \quad \text { for all } \quad f \in \mathcal{H}(G, K), v \in V^{K} .
$$

If $\pi$ is irreducible, then $\pi_{K}$ is an algebraically (respectively, topologically) irreducible representation of $\mathcal{H}(G, K)$. Moreover, every algebraically (respectively, topologically) irreducible representation of $\mathcal{H}(G, K)$ is of the form $\pi_{K}$ for some irreducible smooth (respectively, unitary) representation $\pi$ of $G$.

Proposition 17. Let $G$ be a totally disconnected locally compact group.

(i) Assume that every irreducible unitary representation of $G$ is admissible. Then $G$ is trace class.

(ii) Assume that $G$ has a uniformly admissible smooth dual. Then $G$ has a uniformly admissible unitary dual.

(iii) Assume that $G$ has a uniformly admissible unitary dual. Then every compact open subgroup of $G$ is large.

Proof. To show item (i), observe that the algebra $C_{c}^{\infty}(G)$ of test functions on $G$ mentioned in the introduction is the union $\bigcup_{K} \mathcal{H}(G, K)$, where $K$ runs over the compact open subgroups of $G$. Let $K$ be such a subgroup and $(\pi, V)$ an irreducible 
unitary representation of $G$. Since $\pi\left(e_{K}\right)$ is the orthogonal projection on $V^{K}$ and since $V^{K}$ is finite dimensional, $\pi(f)=\pi\left(e_{K}\right) \pi(f) \pi\left(e_{K}\right)$ has finite rank, for every $f \in \mathcal{H}(G, K)$; see also DvD16, Theorem 2.3].

Item (ii) is proved in [FR19, Theorem B].

To show item (iii), let $K$ be a compact open subgroup of $G$ and let $\sigma \in \widehat{K}$. Let $(\pi, \mathcal{H})$ be an irreducible representation of $G$. Since $K$ is a totally disconnected compact group, there exists a normal open subgroup $L=L(\sigma)$ of $K$ such that $\left.\sigma\right|_{L}$ is the identity representation. By assumption, there exists an integer $N(L)$ such that $\operatorname{dim} \mathcal{H}^{L} \leq N(L)$ for every $(\pi, \mathcal{H}) \in \widehat{G}$. Since

$$
m\left(\sigma,\left.\pi\right|_{K}\right) \operatorname{dim} \sigma \leq \operatorname{dim} \mathcal{H}^{L},
$$

the claim follows.

The following immediate corollary is a useful criterion for the trace class property in the totally disconnected case.

Corollary 18. A totally disconnected locally compact group $G$ is trace class if and only if $G$ is $C C R$.

Proof. As already mentioned, $G$ being trace class implies that $G$ is CCR. Conversely, assume that $G$ is CCR. Then, for every compact open subgroup $K$ and every irreducible representation $\pi$ of $G$, the projection $\pi\left(e_{K}\right)$ is a compact operator and has therefore finite rank. Hence, every irreducible unitary representation of $G$ is admissible and $G$ is trace class by Proposition 17.(i).

As mentioned in the introduction, it is known that reductive algebraic groups over local fields are of type I. In fact, the following stronger result holds.

\section{Theorem 19.}

(i) (Harish-Chandra) Let $G$ be a connected reductive Lie group with finite center. Then every maximal compact subgroup of $G$ is uniformly large in $G$.

(ii) (Bernstein) Let $G=\mathbf{G}(\mathbf{k})$, where $\mathbf{G}$ is a reductive linear algebraic group $\mathbf{G}$ over a non archimedean local field $\mathbf{k}$. Then $G$ has a uniformly admissible smooth dual and hence a uniformly admissible unitary dual.

Every group $G$ as in (i) or (ii) is trace class.

Proof. For (i), we refer to [HC53]. Item (ii) follows from Ber74] in combination with results from [HC70]. Alternatively, Item (ii) is a direct consequence of [Ber74], of the fact that $G$ has an admissible smooth dual (Ber92]), and of Proposition 17 (ii).

The last statement follows from Propositions 15 and 17.

2.6. Covering groups of reductive groups. Let $\mathbf{k}$ be a local field and $\mathbf{G}$ a reductive linear algebraic group defined over $\mathbf{k}$. When $\mathbf{k}$ is archimedean, every finite covering group of $G=\mathbf{G}(\mathbf{k})$ is of type I (see Theorem 19] (i)). We need to show that this result also holds in the non archimedean case, at least for certain finite covers of $G$.

As the following example shows, a central finite extension of a locally compact group of type I is in general not of type I. 
Example 20. Let $\mathbf{F}_{p}$ be the field of order $p$ for a prime $p \geq 3$. Let $V$ be the vector space over $\mathbf{F}_{p}$ of sequences $\left(x_{n}\right)_{n \in \mathbf{N}}$ with $x_{n} \in \mathbf{F}_{p}$ and $x_{n}=0$ for almost all $n \in \mathbf{N}$, and let $\omega$ be the symplectic form on $V \oplus V$, defined by

$$
\omega\left((x, y),\left(x^{\prime}, y^{\prime}\right)\right)=\sum_{n \in \mathbf{N}}\left(x_{n} y_{n}^{\prime}-y_{n} x_{n}^{\prime}\right) \text { for }(x, y),\left(x^{\prime}, y^{\prime}\right) \in V \oplus V .
$$

Let $H_{\infty}\left(\mathbf{F}_{p}\right)$ be the discrete group with underlying set $V \oplus V \oplus \mathbf{F}_{p}$ and with multiplication defined by

$$
(x, y, z)\left(x^{\prime}, y^{\prime}, z^{\prime}\right)=\left(x+x^{\prime}, y+y^{\prime}, z+z^{\prime}+\omega\left((x, y),\left(x^{\prime}, y^{\prime}\right)\right)\right)
$$

for all $(x, y, z),\left(x^{\prime}, y^{\prime}, z^{\prime}\right) \in H_{\infty}\left(\mathbf{F}_{p}\right)$.

The "Heisenberg group" $H_{\infty}\left(\mathbf{F}_{p}\right)$ is a finite central cover of the abelian group $V \oplus V$. It is easily checked that $H_{\infty}\left(\mathbf{F}_{p}\right)$ is not virtually abelian. So, $H_{\infty}\left(\mathbf{F}_{p}\right)$ is not of type I, by Thoma's characterization of discrete groups of type I ([Tho68]).

Let $\mathbf{k}$ be a non archimedean local field, $\mathbf{G}$ a reductive linear algebraic group defined over $\mathbf{k}$, and $G=\mathbf{G}(\mathbf{k})$.

Let $\widetilde{G}$ be a finite central extension of $G$, that is, there exist a finite normal subgroup $F$ contained in the center of $\widetilde{G}$ and a continuous surjective homomorphism $p: \widetilde{G} \rightarrow G$ with $\operatorname{ker} p=F$.

Let $P$ be a parabolic subgroup of $G$. The subgroup $\widetilde{P}=p^{-1}(P)$ is called a parabolic subgroup of $\widetilde{G}$. Let $N$ be the unipotent radical of $P$ and $P=M N$ a Levi decomposition. There exists a unique closed subgroup $\underline{N}$ of $\widetilde{G}$ such that $\left.p\right|_{N}: \underline{N} \rightarrow N$ is an isomorphism (see [Duf82, 11 Lemme]). By its uniqueness property, $\underline{N}$ is a normal subgroup of $\widetilde{P}$, called the unipotent radical of $\widetilde{P}$, and we have a Levi decomposition $\widetilde{P}=\widetilde{M} \underline{N}$, where $\widetilde{M}=p^{-1}(M)$.

We recall a few facts about the structure of $G=\mathbf{G}(\mathbf{k})$ from BT72.

Let $P_{0}$ be a fixed minimal parabolic subgroup of $G$ with unipotent radical $N_{0}$ and Levi decomposition $P_{0}=M_{0} N_{0}$, where $N_{0}$ is the unipotent radical of $P_{0}$. Let $A_{0}$ be a maximal split torus contained in $P_{0}$. For any root $\alpha$ of $A_{0}$, let $\xi_{\alpha}$ be the corresponding character of $A_{0}$ and let $A_{0}^{+}$be the set of all $a \in A_{0}$ with $\left|\xi_{\alpha}(a)\right| \geq 1$. Let $Z$ be a maximal split torus contained in the center of $G$. Let $P_{0}^{--}$be the opposite parabolic subgroup to $P_{0}$ and $N_{0}^{-}$the unipotent radical of $P_{0}^{-}$.

There exists a compact maximal subgroup $K_{0}$ of $G$ such that $G=K_{0} P_{0}$ (Iwasawa decomposition). We have a Cartan decomposition

$$
G=K_{0} S Z \Omega K_{0},
$$

where $S$ is a finitely generated semigroup contained in $A_{0}^{+}$and $\Omega$ is a finite subset of $G$; moreover, the following holds: every neighbourhood of $e$ contains a compact open subgroup $K$ with the following properties:

(i) $K \subset K_{0}$ and $K_{0}$ normalizes $K$;

(ii) We have $K=K^{+} K^{-}$, where $K^{+}=K \cap P_{0}$ and $K^{-}=K \cap N_{0}^{-}$;

(iii) $a^{-1} K^{+} a \subset K^{+}$and $a K^{-} a^{-1} \subset K^{-}$for every $a \in S$.

For all this, see BT72.

We assume from now on that $\widetilde{G}$ satisfies the following Condition $(*)$ :

(*) For every $g, h \in \widetilde{G}$, we have

$$
g h=h g \Longleftrightarrow p(g) p(h)=p(h) p(g) .
$$


Set

$$
\widetilde{K_{0}}=p^{-1}\left(K_{0}\right), \quad \widetilde{S}=p^{-1}(S), \quad \widetilde{Z}=p^{-1}(Z), \quad \text { and } \quad \widetilde{\Omega}=p^{-1}(\Omega) .
$$

Let $\underline{N_{0}}$ and $\underline{N_{0}^{-}}$be the unique subgroups of $\widetilde{G}$ corresponding to $N_{0}$ and $N_{0}^{-}$.

Proposition 21. We have

(1) $\widetilde{G}=\widetilde{K}_{0} \widetilde{S} \widetilde{Z} \widetilde{\Omega} \widetilde{K}_{0}$

(2) $\widetilde{Z}$ is contained in the center of $G$;

(3) $\widetilde{S}$ is a finitely generated commutative semigroup of $\widetilde{G}$;

(4) every neighbourhood of e in $\widetilde{G}$ contains a compact open subgroup $L$ with the following properties:

(4.i) $L \subset \widetilde{K_{0}}$ and $\widetilde{K_{0}}$ normalizes $L$;

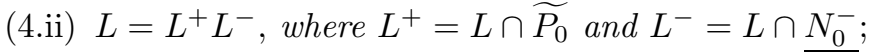

(4.iii) $\widetilde{a}^{-1} L^{+} \widetilde{a} \subset L^{+}$and $\widetilde{a} L^{-} \widetilde{a}^{-1} \subset L^{-}$for every $\widetilde{a} \in \widetilde{S}$.

Proof. It is clear that property (1) holds. It follows from Condition $(*)$ that $\widetilde{Z}$ is contained in the center of $\widetilde{G}$ and that $\widetilde{S}$ is commutative. Moreover, $\widetilde{S}$ is a finitely generated commutative semigroup of $\widetilde{G}$, since $\widetilde{G}$ is a finite covering of $G$. So, properties (2) and (3) are satisfied.

Let $\widetilde{U}$ be an open neighbourhood of $e$ in $\widetilde{G}$ and set $U:=p(\widetilde{U})$. We may assume that $\widetilde{U} \cap F=\{e\}$, where $F=\operatorname{ker} p$; so,

$$
\left.p\right|_{\widetilde{U}}: \widetilde{U} \rightarrow U
$$

is a homeomorphism and we can therefore identify $p^{-1}(U)$ with $U \times F$ and $\widetilde{U}$ with the open open subset $U \times\{e\}$ of $U \times F$.

Let $\widetilde{a_{1}}, \ldots, \widetilde{a_{n}}$ be a generating set of $\widetilde{S}$. Choose a neighborhood $V$ of $e$ contained in $U$ such that $V^{-1}=V, V^{2} \subset U$, and

$$
\widetilde{a_{i}}(V \times\{e\}) \widetilde{a}_{i}^{-1} \subset U \times\{e\} \quad \text { and } \quad \tilde{a}_{i}^{-1}(V \times\{e\}) \widetilde{a_{i}} \subset U \times\{e\} .
$$

for every $i \in\{1, \ldots, n\}$.

Fix a compact open subgroup $K_{1}$ contained in $V$. We can identify $p^{-1}\left(K_{1}\right)$ with $K_{1} \times F$, as topological groups. Observe that $K_{1} \times\{e\}$ is an open subgroup of the compact group $\widetilde{K_{0}}$ and has therefore finite index in $\widetilde{K_{0}}$. Hence, there exists a subgroup of finite index $K_{2}$ of $K_{1}$ such that $K_{2} \times\{e\}$ is normal in $\widetilde{K_{0}}$.

Let $K$ be a compact open subgroup of $K_{0}$ contained in $K_{2}$ and with the properties (i), (ii), and (iii) as above. Set

$$
L:=K \times\{e\} \quad \text { and } \quad L^{+}:=L \cap \widetilde{P_{0}}, \quad L^{-}:=L \cap \underline{N_{0}^{-}} .
$$

Since $K$ is normal in $K_{0}$ and since $L$ is contained in the normal subgroup $K_{2} \times\{e\}$ of $\widetilde{K_{0}}=p^{-1}\left(K_{0}\right)$, it is clear that $L$ is normal in $\widetilde{K_{0}}$.

Moreover, as $K=K^{+} K^{-}$for $K^{+}=K \cap P_{0}$ and $K^{-}=K \cap N_{0}^{-}$, we have $L=L^{+} L^{-}$. So, properties (4.i) and (4.ii) are satisfied. Property (4.iii) is also satisfied. Indeed, let $i \in\{1, \ldots, n\}$ and $a_{i}=p\left(\widetilde{a_{i}}\right)$. On the one hand, we have

$$
p\left(\widetilde{a_{i}}{ }^{-1} L^{+} \widetilde{a_{i}}\right)=a_{i}^{-1} K^{+} a_{i} \subset K^{+}=p\left(L^{+}\right)
$$

and

$$
p\left(\widetilde{a}_{i} L^{-}{\widetilde{a_{i}}}^{-1}\right)=a_{i} K^{-} a_{i}{ }^{-1} \subset K^{-}=p\left(L^{-}\right) .
$$


On the other hand, since $L$ is contained in $V \times\{e\}$, we have

$$
{\widetilde{a_{i}}}^{-1} L^{+} \widetilde{a_{i}} \subset U \times\{e\} \text { and }{\widetilde{a_{i}}}^{-}{\widetilde{a_{i}}}^{-1} \subset U \times\{e\}
$$

and the claim follows.

It is known (see [Ber92, Theorem 15]) that every irreducible smooth representation of $G$ is admissible; the following proposition extends this result to the central cover $\widetilde{G}$. The proof depends on an analysis of cuspidal representations of $\widetilde{G}$.

Let $\widetilde{P}$ be a parabolic subgroup of $\widetilde{G}$ with Levi decomposition $\widetilde{P}=\widetilde{M} \underline{N}$, as defined above.

Let $(\sigma, W)$ be a smooth representation of $\widetilde{M}$. Since $\widetilde{P} / \underline{N}=\widetilde{M}$, the representation $\sigma$ extends to a unique representation of $\widetilde{P}$ which is trivial on $\underline{N}$. One defines the induced representation $\operatorname{Ind}_{\widetilde{P}}^{\widetilde{G}} \sigma$ to be the right regular representation of $\widetilde{G}$ on the vector space $V$ of all locally constant functions $\widetilde{G} \rightarrow W$ with $f(p g)=\sigma(p) f(g)$ for all $g \in \widetilde{G}$ and $p \in \widetilde{P}$.

Let $(\pi, V)$ be a smooth representation of $\widetilde{G}$. Let $V(\underline{N})$ be the $\pi(\widetilde{M})$-invariant subspace generated by $\{\pi(n) v-v \mid n \in \underline{N}, v \in V\}$; so, a smooth representation $\pi_{\underline{N}}$ of $\widetilde{M}$ is defined on $V_{\underline{N}}:=V / V(\underline{N})$.

An irreducible smooth representation $(\pi, V)$ of $\widetilde{G}$ is called cuspidal if $V_{\underline{N}}=\{0\}$, for all unipotent radicals $\underline{N}$ of proper parabolic subgroups of $\widetilde{G}$.

Proposition 22. Every irreducible smooth representation of $\widetilde{G}$ is admissible.

Proof. The proof is along the lines given for $G=\mathbf{G}(\mathbf{k})$ in [Ber92] or [Ren10].

- First step: Let $(\pi, V)$ be a smooth cuspidal representation of $\widetilde{G}$. Then the matrix coefficients of $\pi$ (that is, the functions $g \mapsto v^{*}(\pi(g) v)$, for $v \in V$ and $\left.v^{*} \in V^{*}\right)$ are compactly supported modulo the center of $\widetilde{G}$.

This follows by an adaptation of the proof of the corresponding result for $G$ as in [Ber92, Theorem 14] or [Ren10, §VI.2].

- Second step: Let $(\pi, V)$ be a smooth cuspidal representation of $\widetilde{G}$. Then $\pi$ is admissible.

This is the same proof as in the case of $G$ (see [Ber92, Corollary p.53] or Ren10, $\S \mathrm{VI} .2]$.

- Third step: Let $(\pi, V)$ be a smooth irreducible representation of $\widetilde{G}$. Then there exists a parabolic subgroup $\widetilde{P}=\widetilde{M} \underline{N}$ and a cuspidal representation $\sigma$ of $\widetilde{M}$ such that $\pi$ is isomorphic to a subrepresentation of $\operatorname{Ind}_{\widetilde{P}}^{\widetilde{G}} \sigma$.

The proof is identical to the proof in the case of $G$ as in [Ber92, Lemma 17] or [Ren10, Corollaire p.205].

- Fourth step: Let $(\pi, V)$ be a smooth irreducible representation of $\widetilde{G}$. Then $\pi$ is admissible.

By the third step, we may assume that $\pi$ is a subrepresentation of $\operatorname{Ind}_{\widetilde{P}}^{\widetilde{G}} \sigma$ for some parabolic subgroup $\widetilde{P}=\widetilde{M} \underline{N}$ and a cuspidal representation $\sigma$ of $\widetilde{M}$. By the second step, $\sigma$ is admissible. Since $\widetilde{G} / \widetilde{P}$ is compact, it is easily seen that $\operatorname{Ind}_{\widetilde{P}}^{\widetilde{G}} \sigma$ is admissible (see [Ren10, Lemma III.2.3]). It follows that $\pi$ is admissible.

Corollary 23. Let $\mathbf{G}$ be a reductive linear algebraic group over a non archimedean local field $\mathbf{k}$. Let $\widetilde{G}$ be a central finite cover of $G=\mathbf{G}(\mathbf{k})$ satisfying Condition (*) as above. Then $\widetilde{G}$ is trace class. 
Proof. Let $L$ be a compact open subgroup of $\widetilde{G}$. Proposition 21 shows that Assertion A from Ber74] is satisfied for the totally disconnected locally compact group $\widetilde{G}$. Hence, there exists an integer $N(L) \geq 1$ such that $\operatorname{dim} V^{L} \leq N(L)$ for every admissible smooth representation $(\pi, V)$ of $\widetilde{G}$. Since, by Proposition 22 , every smooth representation of $\widetilde{G}$ is admissible, it follows that $\widetilde{G}$ has a uniformly admissible smooth dual. Proposition 17.(ii) shows that $\widetilde{G}$ is therefore trace class.

The metapletic group $\widetilde{\operatorname{Sp}_{2 n}(\mathbf{k})}$ satisfies Condition $(*)$ as above (see MVW87, Corollaire p. 38]). The following result is therefore a direct consequence of Corollary 23.

Corollary 24. Let $\mathbf{G}$ be a reductive algebraic subgroup of $S p_{2 n}$ and let $G=\mathbf{G}(\mathbf{k})$. Let $\widetilde{G}$ be the inverse image of $G$ in $\widetilde{S p_{2 n}(\mathbf{k})}$. Then $\widetilde{G}$ is a trace class group.

\section{Proofs}

3.1. Proof of Theorem 2. Let $\mathbf{G}^{0}$ be the connected component of $\mathbf{G}$. Since $\mathbf{G}^{0}(\mathbf{k})$ is a normal subgroup of finite index in $\mathbf{G}(\mathbf{k})$, in view of Proposition 6, it suffices to prove the claim when $\mathbf{G}$ is connected.

Let $\mathbf{U}$ be the unipotent radical of $\mathbf{G}$ and $\mathfrak{u}$ its Lie algebra. Through a series of reduction steps, we will be lead eventually to the case where $\mathbf{U}$ is a Heisenberg group.

If $\mathfrak{u}=\{0\}$, then $\mathbf{G}$ is reductive and the claim follows from Dix57 and Ber74.

- First reduction step: we assume from now on that $\mathbf{G}$ is connected and that $\mathfrak{u} \neq\{0\}$.

We proceed by induction on $\operatorname{dim} \mathbf{G}$. So, assume that $\operatorname{dim} \mathbf{G}>0$ and the claim is proved for every connected algebraic group defined over $\mathbf{k}$ with dimension strictly smaller than $\operatorname{dim} \mathbf{G}$.

Let $\rho$ be a factor representation of $G:=\mathbf{G}(\mathbf{k})$, fixed in the sequel. We have to show that $\rho$ is of type $\mathrm{I}$.

Let $\mathfrak{m}$ be a non-zero $\mathbf{G}$-invariant abelian ideal of $\mathfrak{u}$. (An example of such an ideal is the center of $\mathfrak{u}$.) Then $\mathbf{M}=\exp (\mathfrak{m})$ is a non-trivial abelian algebraic normal subgroup of $\mathbf{G}$ contained in $\mathbf{U}$. By Lemma 10, $M:=\mathbf{M}(\mathbf{k})$ is regularly embedded in $G$. Hence, by Theorem [5.(ii), there exists $\chi \in \widehat{M}$ and a factor representation $\pi$ of $G_{\chi}$ such that $\left.\pi\right|_{M}$ is a multiple of $\chi$ and such that $\rho=\operatorname{Ind}_{G_{\chi}}^{G} \pi$.

Recall that $\mathbf{G}$ acts k-rationally by the co-adjoint action of $\mathbf{G}$ on $\mathfrak{m}^{*}$ and that $\chi$ is defined by a unique linear functional $f_{\mathfrak{m}} \in \mathfrak{m}^{*}$ (see Subsection 2.3).

Assume that either $\operatorname{dim} \mathfrak{m} \geq 2$ or $\operatorname{dim} \mathfrak{m}=1$ and $f_{\mathfrak{m}}=0$. In both cases, there exists a non-zero subspace $\mathfrak{k}$ of $\mathfrak{m}$ contained in $\operatorname{ker}\left(f_{\mathfrak{m}}\right)$. Let $\mathbf{K}$ be the corresponding algebraic normal subgroup of $\mathbf{G}$. Then $\rho$ factorizes through the group of k-points of the connected algebraic group $\mathbf{G} / \mathbf{K}$ and $\operatorname{dim} \mathbf{G} / \mathbf{K}<\operatorname{dim} \mathbf{G}$. Hence, $\rho$ is of type I, by the induction hypothesis.

- Second reduction step: we assume from now on that $\mathfrak{u}$ contains no G-invariant abelian ideal $\mathfrak{m}$ with $\operatorname{dim} \mathfrak{m} \geq 2$ or with $\operatorname{dim} \mathfrak{m}=1$ and such that $f_{\mathfrak{m}}=0$. In particular, we have $\operatorname{dim} \mathfrak{z}=1$ and $f_{\mathfrak{z}} \neq 0$, where $\mathfrak{z}$ is the center of $\mathfrak{u}$.

Set $\mathbf{Z}=\exp (\mathfrak{z})$ and set $Z=\mathbf{Z}(\mathbf{k})$. Let $\chi \in \widehat{Z}$ be the character corresponding to $f_{\mathfrak{z}}$ and let $\pi$ be a factor representation of $G_{\chi}$ such that $\left.\pi\right|_{Z}$ is a multiple of $\chi$ and such that $\rho=\operatorname{Ind}_{G_{\chi}}^{G} \pi$. 
Assume that $\operatorname{dim} \mathbf{G}_{\chi}<\operatorname{dim} \mathbf{G}$. Then, by induction hypothesis, the group $\mathbf{G}_{\chi}^{0}(\mathbf{k})$ of the k-points of the connected component of $\mathbf{G}_{\chi}$ is of type I. Since $\mathbf{G}_{\chi}^{0}(\mathbf{k})$ has finite index in $G_{\chi}$, it follows from Proposition 6 that $G_{\chi}$ is of type I. Hence, $\rho$ is of type I by Theorem 5 (i).

- Third reduction step: we assume from now on that $\operatorname{dim} \mathbf{G}_{\chi}=\operatorname{dim} \mathbf{G}$ for $\chi \in \widehat{Z}$ as above. Then $\mathbf{G}_{\chi}$ has finite index in $\mathbf{G}$ and hence $\mathbf{G}_{\chi}=\mathbf{G}$, since $\mathbf{G}$ is connected. So, $G_{\chi}=G$ and in particular $\rho=\pi$.

Observe that, since $\chi \neq 1_{Z}$ and since $\operatorname{dim} \mathbf{Z}=1$, it follows that $\mathbf{G}$ acts trivially on $\mathbf{Z}$. We claim that $\mathfrak{u}$ is not abelian. Indeed, otherwise, we would have $\mathfrak{u}=\mathfrak{z}$ and $\mathbf{G}$ would act trivially on $\mathfrak{u}$; so, $\mathbf{G}$ would be reductive, in contradiction to the first reduction step.

We claim that $\operatorname{dim}[\mathfrak{u}, \mathfrak{u}]=1$. Indeed, assume, by contradiction, that $\operatorname{dim}[\mathfrak{u}, \mathfrak{u}] \geq 2$. Then, setting $\mathfrak{u}^{\prime}:=[\mathfrak{u}, \mathfrak{u}]$, there exist ideals $\mathfrak{u}_{1}, \mathfrak{u}_{2}$ in $\mathfrak{u}$ of dimension 1 and 2 such that $\mathfrak{u}_{1} \subset \mathfrak{u}_{2} \subset \mathfrak{u}^{\prime}$ and $\left[\mathfrak{u}, \mathfrak{u}_{2}\right] \subset \mathfrak{u}_{1}$. Since

$$
\left[\mathfrak{u}^{\prime}, \mathfrak{u}_{2}\right] \subset\left[\left[\mathfrak{u}, \mathfrak{u}_{2}\right], \mathfrak{u}\right]+\left[\left[\mathfrak{u}_{2}, \mathfrak{u}\right], \mathfrak{u}\right] \subset\left[\mathfrak{u}_{1}, \mathfrak{u}\right]=0,
$$

the center $\mathfrak{z}^{\prime}$ of $\mathfrak{u}^{\prime}$ is of dimension $\geq 2$. As $\mathfrak{z}^{\prime}$ is a characteristic ideal, $\mathfrak{z}^{\prime}$ is $\mathbf{G}$-invariant and this is a contradiction to the second reduction step.

In view of Lemma 11, we can state our last reduction step.

- Fourth reduction step: we assume from now on that $\mathbf{U}$ is the Heisenberg group $H_{2 n+1}$ for some $n \geq 1$ and that $\chi \neq 1_{Z}$.

By Theorem 13. (i), there exists a unique irreducible representation $\pi_{\chi}$ of $H_{2 n+1}(\mathbf{k})$ such that $\left.\pi_{\chi}\right|_{Z}$ is a multiple of $\chi$.

Let $\mathbf{L}$ be a Levi subgroup of $\mathbf{G}$ defined over $\mathbf{k}$. So, $\mathbf{L}$ is reductive and $\mathbf{G}=\mathbf{L} \ltimes \mathbf{U}$. By Proposition 12, the action of $G$ on $H_{2 n+1}(\mathbf{k})$ by conjugation gives rise to a continuous homomorphism $\varphi: G \rightarrow S p_{2 n}(\mathbf{k}) \ltimes H_{2 n+1}(\mathbf{k})$.

The representation $\pi_{\chi}$ of $H_{2 n+1}(\mathbf{k})$ extends to an $\omega \circ(p \times p)$-representation $\widetilde{\pi_{\chi}}$ of $S p_{2 n}(\mathbf{k}) \ltimes H_{2 n+1}(\mathbf{k})$ for some $\omega \in Z^{2}\left(S p_{2 n}(\mathbf{k}), \mathbf{T}\right)$, where $p: S p_{2 n}(\mathbf{k}) \ltimes H_{2 n+1}(\mathbf{k}) \rightarrow$ $S p_{2 n}(\mathbf{k})$ is the canonical projection. By Theorem 13.(ii), one can choose $\omega$ so that the image of $\omega$ is $\{ \pm 1\}$ and the corresponding central extension of $S p_{2 n}(\mathbf{k})$ is the metaplectic group $\widetilde{S p_{2 n}(\mathbf{k})}$. Setting

$$
\omega^{\prime}:=\omega \circ(p \times p) \circ(\varphi \times \varphi) \in Z^{2}(G, \mathbf{T})
$$

and $\widetilde{\pi}^{\prime}:=\widetilde{\pi_{\chi}} \circ \varphi$, we have that $\widetilde{\pi}^{\prime}$ is an $\omega^{\prime}$-representation of $G$ which extends $\pi_{\chi}$.

Let $\sigma$ be a factor $\omega^{\prime}$-representation of $L=\mathbf{L}(\mathbf{k})$ lifted to $G$. (Observe that $\omega^{\prime-1}=\omega^{\prime}$.) Let $\widetilde{L}=\{ \pm 1\} \times L$ be the central extension of $L$ defined by $\omega^{\prime}$.

We claim that $\widetilde{L}$ satisfies Condition $(*)$ as before Proposition 21. Indeed, let $x, y \in L$ be such that $x y=y x$; then $\varphi(x) \varphi(y)=\varphi(y) \varphi(x)$ and hence

$$
\omega^{\prime}(x, y)=\omega(\varphi(x), \varphi(y))=\omega(\varphi(y), \varphi(x))=\omega^{\prime}(y, x),
$$

since Condition $(*)$ holds for $\widetilde{S p_{2 n}(\mathbf{k})}$. This proves the claim.

It follows from Corollary 23 that $\widetilde{L}$ is of type I. Theorem (iii) then implies that the factor representation $\rho$ of $G$ is of type $I$.

3.2. Proof of Theorem 4. As mentioned before, the fact that (i) implies (ii) was already shown by Lipsman in [Lip75, Theorem 3.1 and Lemma 4.1].

We claim that (ii) implies (i). Indeed, let $\mathbf{M}$ be as in Theorem 4, Then $\mathbf{M}$ is an algebraic normal subgroup of the reductive group $\mathbf{L}$ and is therefore reductive as 
well (see Bor91, Corollary in $\S 14.2]$ ). Since $\mathbf{M}(\mathbf{k})$ commutes with all elements in $\mathbf{U}(\mathbf{k})$, it follows that

$$
N:=\mathbf{M}(\mathbf{k}) \mathbf{U}(\mathbf{k}) \cong \mathbf{M}(\mathbf{k}) \times \mathbf{U}(\mathbf{k})
$$

is a direct product of groups. Now, $\mathbf{M}(\mathbf{k})$ and $\mathbf{U}(\mathbf{k})$ are CCR (even trace class) by the results mentioned in the introduction. Hence, $N$ is CCR as well (and by DvD16, Proposition 1.9] even trace class). Since $\mathbf{G}(\mathbf{k}) / N \cong \mathbf{L}(\mathbf{k}) / \mathbf{M}(\mathbf{k})$ is compact, it follows from a general result (see [Sch70, Proposition 4.3]) that $\mathbf{G}(\mathbf{k})$ is CCR.

It remains to show that (ii) implies (iii).

If $\mathbf{k}$ is non-archimedean then $G=G(\mathbf{k})$ is totally disconnected and CCR and it follows from Corollary 18 that $G$ is trace class. The proof in the archimedean case is more involved.

Assume that $\mathbf{k}=\mathbf{R}$ and that (ii) holds. Let

$$
N:=\mathbf{M}(\mathbf{R}) \mathbf{U}(\mathbf{R}) \cong \mathbf{M}(\mathbf{R}) \times \mathbf{U}(\mathbf{R})
$$

be as above. We already observed above that $N$ is trace class and that $G / N$ is compact, where $G=\mathbf{G}(\mathbf{R})$. Note that $N$ is unimodular, since $\mathbf{M}(\mathbf{R})$ and $\mathbf{U}(\mathbf{R})$ are unimodular. Since, moreover, $G / N$ is compact, it follows from Weil's formula

$$
\int_{G} f(g) d g=\int_{G / N} \int_{N} f(g n) d n d \dot{g}, \quad f \in C_{C}(G)
$$

that $G$ is unimodular as well.

Since subrepresentations of trace class representations are trace class and in view of Corollary 7, it suffices to show that, for every $\pi \in \widehat{N}$, the induced representation $\rho=\operatorname{Ind}_{N}^{G} \pi$ is a (not necessarily irreducible) trace class representation of $G$, i.e., the operator $\rho(f)$ is a trace-class operator for all $f \in C_{c}^{\infty}(G)$.

Recall that, since $G / N$ is compact and both $G$ and $N$ are unimodular, the Hilbert space $\mathcal{H}_{\rho}$ for the induced representation $\rho=\operatorname{Ind}_{N}^{G} \pi$ is a completion of the vector space

$$
\mathcal{F}_{\rho}=\left\{\xi \in C\left(G, \mathcal{H}_{\pi}\right): \xi(g n)=\pi\left(n^{-1}\right) \xi(g) \quad \forall g \in G \forall n \in N\right\},
$$

with inner product given by $\langle\xi, \eta\rangle=\int_{G / N}\langle\xi(g), \eta(g)\rangle d \dot{g}$. The induced representation $\rho=\operatorname{Ind}_{N}^{G} \pi$ acts on the dense subspace $\mathcal{F}_{\rho}$ by the formula $(\rho(s) \xi)(g)=\xi\left(s^{-1} g\right)$.

Now let $f \in C_{c}^{\infty}(G)$. Following some ideas in the proof of Sch70, Proposition 4.2], we compute for $\xi \in \mathcal{F}_{\rho}$ :

$$
\begin{aligned}
(\rho(f) \xi)(g) & =\int_{G} f(s) \xi\left(s^{-1} g\right) d s \stackrel{s \mapsto \underline{g s}^{-1}}{=} \int_{G} f\left(g s^{-1}\right) \xi(s) d s \\
& =\int_{G / N} \int_{N} f\left(g n^{-1} s^{-1}\right) \xi(s n) d n d \dot{s} \\
& \stackrel{n \mapsto n^{-1}}{=} \int_{G / N} \int_{N} f\left(g n s^{-1}\right) \xi\left(s n^{-1}\right) d n d \dot{s} \\
& =\int_{G / N} \int_{N} f\left(g n s^{-1}\right) \pi(n) \xi(s) d n d \dot{s} \\
& =\int_{G / N} k_{f}(g, s) \xi(s) d \dot{s},
\end{aligned}
$$

where $k_{f}: G \times G \rightarrow \mathcal{B}\left(\mathcal{H}_{\pi}\right)$ is given by $k_{f}(g, s)=\pi\left(\varphi_{f}(g, s)\right)$ and $\varphi_{f}: G \times G \rightarrow$ $C_{c}^{\infty}(N)$ is given by $\varphi_{f}(g, s)(n):=f\left(g n s^{-1}\right)$. 
Since $\pi$ is trace class, the map $k_{f}=\pi \circ \varphi_{f}$ takes its values in the space of traceclass operators. As trace class operators are Hilbert-Schmidt operators, we may regard $k_{f}$ as a map into the set $\mathcal{H} S\left(\mathcal{H}_{\pi}\right)$ of Hilbert-Schmidt operators on $\mathcal{H}_{\pi}$.

We claim that the map $G \times G \rightarrow\left\|k_{f}(g, s)\right\|_{\mathrm{HS}}$ is continuous, where $\|\cdot\|_{\mathrm{HS}}$ denotes the Hilbert-Schmidt norm. This will follow from [DvD16, Proposition 1.4] as soon as we have shown that the map

$$
\varphi_{f}: G \times G \rightarrow C_{c}^{\infty}(N)
$$

is continuous, where $C_{c}^{\infty}(N)$ is equipped with the Fréchet space topology as introduced in DvD16, Definition 1.1]. But this is a consequence of the continuity of the map $C_{c}^{\infty}(G) \rightarrow C_{c}^{\infty}(N),\left.\varphi \mapsto \varphi\right|_{N}$ and of the map $G \times G \rightarrow C_{c}^{\infty}(G),(g, s) \mapsto L_{s} R_{g} f$, where $L_{s}$ (resp. $R_{g}$ ) denote left (resp. right) translation by $s$ (resp. $g$ ).

For $k, m \in N$, we have

$$
\begin{aligned}
k_{f}(g k, s m) & =\int_{N} f\left(g k n m^{-1} s^{-1}\right) \pi(n) d n \\
& \stackrel{n \mapsto k^{-1} n m}{=} \int_{N} f\left(g n s^{-1}\right) \pi\left(k^{-1} n m\right) d n \\
& =\pi\left(k^{-1}\right) k_{f}(g, s) \pi(m) .
\end{aligned}
$$

It follows that the Hilbert-Schmidt norm of $k_{f}(g, s)$ is constant on $N$-cosets in both variables. As $G / N$ is compact, this implies that the continuous map $(g, s) \mapsto$ $\left\|k_{f}(g, s)\right\|_{\text {HS }}$ is bounded.

We want to conclude from this that $\rho(f)$ is a Hilbert-Schmidt operator. For this, let $c: G / N \rightarrow G$ be a Borel section for the quotient map $p: G \rightarrow G / N$. Let $V: \mathcal{H}_{\rho} \rightarrow L^{2}\left(G / N, \mathcal{H}_{\pi}\right)$ be defined on $\mathcal{F}_{\rho}$ by $\xi \mapsto V \xi:=\xi \circ c$. Then it is straightforward to check that $V$ is a unitary operator which intertwines $\rho(f)$ with the integral operator $K_{f}: L^{2}\left(G / N, \mathcal{H}_{\pi}\right) \rightarrow L^{2}\left(G / N, \mathcal{H}_{\pi}\right)$ given by

$$
\left(K_{f} \xi\right)(\dot{g})=\int_{G / N} \tilde{k}_{f}(\dot{g}, \dot{s}) \xi(\dot{s}) d \dot{s}
$$

where $\tilde{k}_{f}(\dot{g}, \dot{s}):=k_{f}(c(\dot{g}), c(\dot{s}))$ for $\dot{g}, \dot{s} \in G / N$.

It suffices to prove that $K_{f}$ is Hilbert-Schmidt. For this, let $\left\{e_{i}: i \in I\right\}$ and $\left\{v_{j}: j \in J\right\}$ be orthonormal bases of $L^{2}(G / N)$ and $\mathcal{H}_{\pi}$, respectively. Observe that $I$ and $J$ are at most countable, since $L^{2}(G / N)$ and $\mathcal{H}_{\pi}$ are separable, by the second countability of $G$.

For a pair $(j, l) \in J \times J$, denote by

$$
k_{j l}(\cdot, \cdot):=\left\langle\tilde{k}_{f}(\cdot, \cdot) v_{j}, v_{l}\right\rangle
$$

the $(j, l)$-th matrix coefficient of $\tilde{k}_{f}$ with respect to $\left\{v_{j}: j \in J\right\}$. Identifying elements $g \in G$ with their images in $G / N$ in the following formulas, we then get

$$
\left\langle\tilde{k}_{f}(g, s) v_{j}, \tilde{k}_{f}(g, t) v_{j}\right\rangle=\sum_{l} k_{j l}(g, s) \overline{k_{j l}(g, t)}
$$


for $g, s, t \in G$. Using this and the fact that $\left\{\bar{e}_{i}: i \in I\right\}$ is also an orthonormal basis of $L^{2}(G / N)$, we compute

$$
\begin{aligned}
\left\|K_{f}\right\|_{\mathrm{HS}}^{2} & =\sum_{i, j}\left\langle K_{f}\left(e_{i} \otimes v_{j}\right), K_{f}\left(e_{i} \otimes v_{j}\right)\right\rangle \\
& =\sum_{i, j} \int_{G / N \times G / N \times G / N}\left\langle\tilde{k}_{f}(g, s) e_{i}(s) v_{j}, \tilde{k}_{f}(g, t) e_{i}(t) v_{j}\right\rangle d(g, s, t) \\
& \stackrel{\underline{\underline{2}}}{=} \sum_{i, j} \int_{G / N \times G / N \times G / N} e_{i}(s) \overline{e_{i}(t)} \sum_{l} k_{j l}(g, s) \overline{k_{j l}(g, t)} d(g, s, t) \\
& =\sum_{i, j, l} \int_{G / N}\left\langle k_{j l}(g, \cdot), \bar{e}_{i}\right\rangle\left\langle\bar{e}_{i}, k_{j l}(g, \cdot)\right\rangle d g \\
& =\sum_{j, l} \int_{G / N}\left\|k_{j l}(g, \cdot)\right\|_{2}^{2} d g \\
& =\sum_{j, l}\left\|k_{j l}\right\|_{2}^{2}=\sum_{j, l} \int_{G / N \times G / N}\left|k_{j l}(g, s)\right|^{2} d(g, s) \\
& \stackrel{\underline{\underline{2}}}{=} \int_{G / N \times G / N} \sum_{j}\left\langle\tilde{k}_{f}(g, s) v_{j}, \tilde{k}_{f}(g, s) v_{j}\right\rangle d(g, s) \\
& =\int_{G / N \times G / N}\left\|\tilde{k}_{f}(g, s)\right\|_{\mathrm{HS}}^{2} d(g, s)<\infty .
\end{aligned}
$$

It follows that $\rho(f)$ is a Hilbert-Schmidt operator for all $f \in C_{c}^{\infty}(G)$, and since $C_{c}^{\infty}(G)=C_{c}^{\infty}(G) * C_{c}^{\infty}(G)$ by [DM78, Theorem 3.1], this implies that $\rho(f)$ is trace class for all $f \in C_{c}^{\infty}(G)$. This finishes the proof.

\section{REFERENCES}

[BdlHV08] Bachir Bekka, Pierre de la Harpe, and Alain Valette, Kazhdan's property (T), New Mathematical Monographs, vol. 11, Cambridge University Press, Cambridge, 2008, DOI 10.1017/CBO9780511542749. MR2415834

[Ber74] I. N. Bernštĕ̌n, All reductive p-adic groups are of type I (Russian), Funkcional. Anal. i Priložen. 8 (1974), no. 2, 3-6. MR0348045

[Ber92] I. N. Bernštel̆n, Draft of: Representations of p-adic groups. (1992), available at http:// www.math.tau.ac.il/ bernstei/. Notes taken by E. Rumelhart.

[Bor91] Armand Borel, Linear algebraic groups, 2nd ed., Graduate Texts in Mathematics, vol. 126, Springer-Verlag, New York, 1991, DOI 10.1007/978-1-4612-0941-6. MR.1102012

[BS64] A. Borel and J.-P. Serre, Théorèmes de finitude en cohomologie galoisienne (French), Comment. Math. Helv. 39 (1964), 111-164, DOI 10.1007/BF02566948. MR181643

[Bru61] François Bruhat, Distributions sur un groupe localement compact et applications à l'étude des représentations des groupes §-adiques (French), Bull. Soc. Math. France 89 (1961), 43-75. MR.140941

[BT72] F. Bruhat and J. Tits, Groupes réductifs sur un corps local (French), Inst. Hautes Études Sci. Publ. Math. 41 (1972), 5-251. MR327923

[DvD16] Anton Deitmar and Gerrit van Dijk, Trace class groups, J. Lie Theory 26 (2016), no. 1, 269-291, DOI 10.1007/s40593-015-0088-2. MR3391353

[Dix57] J. Dixmier, Sur les représentations unitaires des groupes de Lie algébriques (French), Ann. Inst. Fourier (Grenoble) 7 (1957), 315-328. MR99380

[Dix59] Jacques Dixmier, Sur les représentations unitaires des groupes de Lie nilpotents. $V$ (French), Bull. Soc. Math. France 87 (1959), 65-79. MR.115097 
[Dix77] Jacques Dixmier, $C^{*}$-algebras, North-Holland Publishing Co., Amsterdam-New YorkOxford, 1977. Translated from the French by Francis Jellett; North-Holland Mathematical Library, Vol. 15. MR 0458185

[DM78] Jacques Dixmier and Paul Malliavin, Factorisations de fonctions et de vecteurs indéfiniment différentiables (French, with English summary), Bull. Sci. Math. (2) 102 (1978), no. 4, 307-330. MR517765

[Duf82] Michel Duflo, Théorie de Mackey pour les groupes de Lie algébriques (French), Acta Math. 149 (1982), no. 3-4, 153-213, DOI 10.1007/BF02392353. MR688348

[FR19] Uriya A. First and Thomas Rüd, On uniform admissibility of unitary and smooth representations, Arch. Math. (Basel) 112 (2019), no. 2, 169-179, DOI 10.1007/s00013018-1257-y. MR 3908835

[Gli61] J. Glimm, Type I $C^{*}$-algebras, Ann. of Math. (2) 73 (1961), 572-612.

[Fol89] Gerald B. Folland, Harmonic analysis in phase space, Annals of Mathematics Studies, vol. 122, Princeton University Press, Princeton, NJ, 1989, DOI 10.1515/9781400882427. MR983366

[HC53] Harish-Chandra, Representations of a semisimple Lie group on a Banach space. I, Trans. Amer. Math. Soc. 75 (1953), 185-243, DOI 10.2307/1990730. MR56610

[HC70] Harish-Chandra, Harmonic analysis on reductive p-adic groups, Lecture Notes in Mathematics, Vol. 162, Springer-Verlag, Berlin-New York, 1970. Notes by G. van Dijk. MR.0414797

[Kir62] A. A. Kirillov, Unitary representations of nilpotent Lie groups (Russian), Uspehi Mat. Nauk 17 (1962), no. 4 (106), 57-110. MR0142001

[Lip75] Ronald L. Lipsman, The CCR property for algebraic groups, Amer. J. Math. 97 (1975), no. 3, 741-752, DOI 10.2307/2373774. MR390123

[Mac57] George W. Mackey, Les ensembles boréliens et les extensions des groupes (French), J. Math. Pures Appl. (9) 36 (1957), 171-178. MR89998

[Mac58] George W. Mackey, Unitary representations of group extensions. I, Acta Math. 99 (1958), 265-311, DOI 10.1007/BF02392428. MR98328

[MVW87] Colette Mœglin, Marie-France Vignéras, and Jean-Loup Waldspurger, Correspondances de Howe sur un corps p-adique (French), Lecture Notes in Mathematics, vol. 1291, Springer-Verlag, Berlin, 1987, DOI 10.1007/BFb0082712. MR1041060

[Moo65] Calvin C. Moore, Decomposition of unitary representations defined by discrete subgroups of nilpotent groups, Ann. of Math. (2) 82 (1965), 146-182, DOI 10.2307/1970567. MR 181701

[Puk99] Lajos Pukánszky, Characters of connected Lie groups, Mathematical Surveys and Monographs, vol. 71, American Mathematical Society, Providence, RI, 1999. With a preface by J. Dixmier and M. Duflo, DOI 10.1090/surv/071. MR1707323

[Ren10] David Renard, Représentations des groupes réductifs p-adiques (French), Cours Spécialisés [Specialized Courses], vol. 17, Société Mathématique de France, Paris, 2010. MR.2567785

[Sch70] I. Schochetman, Topology and the duals of certain locally compact groups, Trans. Amer. Math. Soc. 150 (1970), 477-489, DOI 10.2307/1995530.

[Tho68] Elmar Thoma, Eine Charakterisierung diskreter Gruppen vom Typ I (German), Invent. Math. 6 (1968), 190-196, DOI 10.1007/BF01404824. MR248288

[vD19] Gerrit van Dijk, Trace class groups: the case of semi-direct products, J. Lie Theory 29 (2019), no. 2, 375-390. MR3915545

[War72] Garth Warner, Harmonic analysis on semi-simple Lie groups. I, Die Grundlehren der mathematischen Wissenschaften, Band 188, Springer-Verlag, New York-Heidelberg, 1972. MR0498999

[Wei64] André Weil, Sur certains groupes d'opérateurs unitaires (French), Acta Math. 111 (1964), 143-211, DOI 10.1007/BF02391012. MR.165033

[Zim84] Robert J. Zimmer, Ergodic theory and semisimple groups, Monographs in Mathematics, vol. 81, Birkhäuser Verlag, Basel, 1984, DOI 10.1007/978-1-4684-9488-4. $\operatorname{MR} 776417$ 
Université Rennes, CNRS, IRMAR-UMR 6625, Campus Beaulieu, F-35042 Rennes Cedex, France

Email address: bachir.bekka@univ-rennes1.fr

Mathematisches Institut,Universität Münster, Einsteinstrasse 62,, D-48149 Münster, Germany

Email address: echters@uni-muenster.de 